\title{
SUPERCONDUCTIVITY VERSUS TUNNELING \\ IN A DOPED ANTIFERROMAGNETIC LADDER
}

\author{
J. P. Rodriguez \\ Instituto de Ciencia de Materiales, Consejo Superior de Investigaciones Científicas, \\ Cantoblanco, 28049 Madrid, Spain and \\ Dept. of Physics and Astronomy, California State University, Los Angeles, CA 90032, USA.*
}

\begin{abstract}
The low-energy charge excitations of a doped antiferromagnetic ladder are modeled by a system of interacting spinless fermions that live on the same ladder. A relatively large spin gap is assumed to "freeze out" all spin fluctuations. We find that the formation of rung hole pairs coincides with the opening of a single-particle gap for charge excitations along chains and with the absence of coherent tunneling in between chains. We also find that such hole pairs condense into either a crystalline or superconducting state as a function of the binding energy.
\end{abstract}

PACS Indices: 74.20.Mn, 75.10.Jm, 74.72.Jt, 74.25.Fy

\footnotetext{
* Permanent address.
} 
One of the strangest features of high-temperature superconductors is the semiconducting nature of electric transport perpendicular to the copper-oxygen planes common to these materials shown in the normal state. ${ }^{1}$ Anderson and co-workers have proposed that this behavior is intrinsic, and that it results from the microscopic coincidence of Cooper pairing with incoherent tunneling in between adjacent metallic planes. ${ }^{2}$ Analogous behavior has been observed recently in the doped "ladder" materials. ${ }^{3}$ In particular, a large anisotropy in the electronic conduction with respect to the ladder direction appears ${ }^{4}$ at compositions that exhibit superconductivity. ${ }^{5,6}$ The authors of ref. 4 suggest that the chain version of the above inter-layer pair/tunneling (IPT) mechanism is responsible for the phenomenon. ${ }^{7,8}$

Such "ladder" materials are made up of a parallel arrangement of magnetically isolated antiferromagnetic ladders that fill copper-oxygen planes similar to those of the cuprate superconductors. They notably show a spin gap on the order of $\Delta_{\text {spin }} \sim 500 \mathrm{~K}$ when undoped, in accord with theoretical expectations based on the spin-1/2 antiferromagnetic Heisenberg ladder. ${ }^{3}$ The actual ladders in these materials can also be doped with a concentration $x$ of mobile holes,${ }^{9}$ in which case the spin gap persists. Again, theoretical studies of the $t-J$ model for a lightly doped ladder, $t x<J$, find evidence for the formation of hole pairs along rungs that leave the remaining singlet bonds along the rungs intact. ${ }^{10}$ The spin gap persists, as a result, and charge excitations are left as the only low-energy excitations.

In this paper, we shall model the low-energy singlet charge excitations of a doped antiferromagnetic ladder by interacting spinless fermions that live on the same ladder in the vicinity of half filling. ${ }^{11}$ We presume, therefore, that spin excitations are frozen out due to a relatively large spin gap of order $\Delta_{\text {spin }} \sim J / 2$. A bosonization analysis based on the corresponding Luther-Emery model, ${ }^{12-15}$ and generalizations thereof, ${ }^{16,17}$ yields that the appearance of hole pairs along rungs coincides with the absence of coherent tunneling in between chains, as well as with the creation of a gap for single-particle excitations along chains. Also, while the hole pairs generally crystallize into a charge density-wave (CDW) state in the weak-coupling limit, ${ }^{3}$ we predict that they Bose condense into an IPT-type superconductor at large enough binding energies (see Fig. 1). Last, the correlations in between pairs that form along the chains are found to be short range when hole pairing along rungs occurs. 
To motivate the spinless fermion analysis that will follow, consider first the standard nearest-neighbor $t-J$ model Hamiltonian

$$
H=\sum_{\langle i, j\rangle}\left[-t\left(\tilde{c}_{i, s}^{\dagger} \tilde{c}_{j, s}+\text { h.c. }\right)+J \vec{S}_{i} \cdot \vec{S}_{j}\right]
$$

for a doped antiferromagnetic ladder. Above, $\tilde{c}_{i, s}^{\dagger}$ creates a spin $s$ electron on site $i$ as long as this site is unoccupied, while $\vec{S}_{i}$ measures the spin at site $i$. At half-filling, the $t-J$ model (1) reduces to the Heisenberg ladder, which is known to have a spin gap $\Delta_{\text {spin }} \cong J / 2 .^{3}$ The latter persists in the presence of a dilute hole concentration, $t x<J .{ }^{10}$ In general, long wavelength electronic excitations within chains can be described via the standard Luther-Emery (LE) model. ${ }^{12-15}$ The spin gap present in lightly doped $t-J$ ladders indicates then that the intra-chain LE backscattering term has the form

$$
H_{\text {back }} \sim-\left(\Delta_{\text {spin }} / a\right) \sum_{l=1}^{2} \int d x \cos \left[\phi_{\uparrow}(x, l)-\phi_{\downarrow}(x, l)\right]
$$

where $\phi_{s}(x, l)$ is the bosonic field that represents collective particle-hole excitations of spin $s$ electrons at position $x=i \cdot a$ along chain $l$ of the ladder. ${ }^{18}$ Yet long-wavelength charge excitations have a typical energy scale on the order of the kinetic energy, $\Delta_{\text {charge }} \sim t x$, which is small in comparison to the spin gap, $\Delta_{\text {spin }}$, in the low doping limit $t x \ll J$. We may therefore take $\Delta_{\text {spin }} \rightarrow \infty$ in this limit. The bosonic spin degrees of freedom are frozen in such case: $\phi_{\uparrow}=\phi=\phi_{\downarrow}$. The remaining (bosonic) charge degree of freedom $\phi(x, l)$ must then correspond to an effective spinless fermion on each chain. This idea is developed below.

In general, the Hamiltonian for a system of $N$ consecutively coupled chains of spinless fermions $^{8}$ can be divided into parallel and perpendicular parts, $H=H_{\|}+H_{\perp}$, where

$$
H_{\|}=\sum_{l=1}^{N} \sum_{i}\left[-t_{\|}\left(f_{i, l}^{\dagger} f_{i+1, l}+\text { h.c. }\right)+V_{\|} n_{i, l} n_{i+1, l}\right]
$$

and

$$
\begin{aligned}
H_{\perp}=\sum_{l=1}^{N} \sum_{i}\left[-t_{\perp}\left(e^{i \Phi / N} f_{i, l}^{\dagger} f_{i, l+1}\right.\right. & + \text { h.c. })+U_{\perp} n_{i, l} n_{i, l+1}+ \\
& \left.+V_{\perp}\left(n_{i, l} n_{i+1, l+1}+n_{i, l+1} n_{i+1, l}\right)\right]
\end{aligned}
$$


describe respectively the quantum mechanics within and in between chains. Here, $f_{i, l}$ denotes the annihilation operator for the spinless fermion on the $i^{\text {th }}$ site of chain $l$, with occupation number $n_{i, l}=f_{i, l}^{\dagger} f_{i, l}$. Also, $t_{\|}$and $t_{\perp}$ are the nearest-neighbor hopping matrix elements, while $V_{\|}, U_{\perp}$ and $V_{\perp}$ are the model interaction energies. In the case of open perpendicular boundary conditions, we shall set $f_{i, N+1}=0=f_{i, N+1}^{\dagger}$. On the other hand, the identifications $f_{i, N+1}=f_{i, 1}$ and $f_{i, N+1}^{\dagger}=f_{i, 1}^{\dagger}$ produce periodic boundary conditions, in which case $\Phi$ denotes the magnetic flux along the parallel direction. Consider now the simple ladder, with $N=2$ chains and open perpendicular boundary conditions $(\Phi=0)$. Since low-energy spin excitations are frozen out due to the formation of singlet bonds along the rungs, ${ }^{3}$ it is natural to identify the true electron field $c_{i, l, s}$ with the spinless fermion field following $f_{i, 1}=c_{i, 1, s(i)}$ and $f_{i, 2}=c_{i, 2,-s(i)}$, where $s(i)$ represents the antiferromagnetic spin configuration on a given chain. After some algebraic manipulations, it can be shown that the above spinless-fermion Hamiltonian takes the form of an extended Hubbard model $^{14}$ in transverse magnetic field:

$$
\begin{aligned}
H=\sum_{i}[ & -t_{\|} \sum_{l=1}^{2}\left(f_{i, l}^{\dagger} f_{i+1, l}+\text { h.c. }\right)-t_{\perp}\left(f_{i, 1}^{\dagger} f_{i, 2}+\text { h.c. }\right)+ \\
& \left.+\left(U_{\perp}+2 V_{\perp}\right) n_{i, 1} n_{i, 2}-\frac{1}{2} V\left(n_{i+1}-n_{i}\right)^{2}-\frac{1}{4} V^{\prime}\left(m_{i+1}-m_{i}\right)^{2}\right],
\end{aligned}
$$

where $n_{i}=n_{i, 1}+n_{i, 2}$ and $m_{i}=n_{i, 2}-n_{i, 1}$, and where $V=\left(V_{\|}+V_{\perp}\right) / 2$ and $V^{\prime}=V_{\|}-V_{\perp}$. It is important to remark that the interaction terms in this ladder model are invariant with respect to $S U(2)$ rotations of the chain labels if $V_{\|}=V_{\perp}$.

We now rotate to the bonding-antibonding basis, $f_{i, \pm}=2^{-1 / 2}\left(f_{i, 2} \pm f_{i, 1}\right)$, that diagonalizes the transverse kinetic energy (5). In the limit near (but not at) half-filling, all umklapp processes are negligible. Taking the continuum limit of the ladder model (5) $\grave{a} l a$ Kogut and Susskind ${ }^{14}$ then yields the Luttinger model $H=H_{\|}^{\prime}+H_{\perp}^{\prime}$, where

$$
H_{\|}^{\prime}=\sum_{n} \int d x\left[2 t_{\|} a\left(L_{n}^{\dagger} i \partial_{x} L_{n}-R_{n}^{\dagger} i \partial_{x} R_{n}\right)+4 V a L_{n}^{\dagger} R_{n}^{\dagger} R_{n} L_{n}-\mu_{n}\left(L_{n}^{\dagger} L_{n}+R_{n}^{\dagger} R_{n}\right)\right] \text {, }
$$

and

$$
H_{\perp}^{\prime}=H_{\perp, 1}^{\prime}+H_{\perp, 2}^{\prime}+H_{\perp, 4}^{\prime}+H_{\perp, \text { pair }}^{\prime}
$$

are rotated parallel and perpendicular pieces, with a backscattering term

$$
H_{\perp, 1}^{\prime}=\sum_{n<n^{\prime}} \int d x\left(U_{\perp}-2 V_{\|}\right) a\left[L_{n}^{\dagger} R_{n^{\prime}}^{\dagger} L_{n^{\prime}} R_{n}+\text { h.c. }\right]
$$


with inter-band forward scattering terms

$$
\begin{aligned}
& H_{\perp, 2}^{\prime}=\sum_{n<n^{\prime}} \int d x 2\left(V_{\|}-V_{\perp}\right) a\left[L_{n}^{\dagger} R_{n^{\prime}}^{\dagger} R_{n^{\prime}} L_{n}+\text { h.c. }\right] \\
& H_{\perp, 4}^{\prime}=\sum_{n<n^{\prime}} \int d x\left(U_{\perp}+2 V_{\perp}\right) a:\left(L_{n}^{\dagger} L_{n}+R_{n}^{\dagger} R_{n}\right)::\left(L_{n^{\prime}}^{\dagger} L_{n^{\prime}}+R_{n^{\prime}}^{\dagger} R_{n^{\prime}}\right):
\end{aligned}
$$

and with an inter-band pseudo-triplet pairing interaction

$$
H_{\perp, \text { pair }}^{\prime}=\sum_{n<n^{\prime}} \int d x 2\left(V_{\|}-V_{\perp}\right) a\left[L_{n}^{\dagger} R_{n}^{\dagger} R_{n^{\prime}} L_{n^{\prime}}+\text { h.c. }\right] .
$$

Here, $e^{i k_{F} x} R_{n}(x)=L^{-1 / 2} \sum_{k} e^{i k x} a_{n}(k)$ and $e^{-i k_{F} x} L_{n}(x)=L^{-1 / 2} \sum_{k} e^{i k x} b_{n}(k)$ denote field operators for right and left moving spinless fermions in the bonding or antibonding band $n=+,-$ for chains of length $L$, with a Fermi surface at $\pm k_{F}$. Above, the symbols ': :' represent normal ordering. ${ }^{14}$ Also, $\mu_{ \pm}= \pm t_{\perp}$ are the chemical potentials for each band. Notice that Eqs. (6)-(10) describe a Luther-Emery model for pseudo spin-1/2 fermions. Since such fermions experience pseudo spin-charge separation, we have that the coupled chains factorize following $H=H_{\rho}+H_{\sigma}$, where

$$
\begin{array}{r}
H_{\rho}=2 \pi \hbar v_{\rho} \sum_{q>0} \sum_{j=R, L} \rho_{j}(q) \rho_{j}(-q)+g_{\rho} \sum_{q} \rho_{R}(q) \rho_{L}(-q) \\
H_{\sigma}=2 \pi \hbar v_{\sigma} \sum_{q>0} \sum_{j=R, L} \sigma_{j}(q) \sigma_{j}(-q)+g_{\sigma} \sum_{q} \sigma_{R}(q) \sigma_{L}(-q)+ \\
+H_{\perp, 1}^{\prime}+H_{\perp, \text { pair }}^{\prime}-(2 L)^{1 / 2} t_{\perp}\left[\sigma_{L}(0)+\sigma_{R}(0)\right]
\end{array}
$$

are the respective commuting portions of the Hamiltonian. Here, $\rho_{j}(q)=2^{-1 / 2}\left[\rho_{j}(q,+)+\right.$ $\left.\rho_{j}(q,-)\right]$ and $\sigma_{j}(q)=2^{-1 / 2}\left[\rho_{j}(q,+)-\rho_{j}(q,-)\right]$ are the standard particle-hole operators for total-charge and pseudo-spin excitations with respect to the bands $n=+,-$, with $\rho_{R}(q, n)=L^{-1 / 2} \sum_{k} a_{n}^{\dagger}(q+k) a_{n}(k)$ and $\rho_{L}(q, n)=L^{-1 / 2} \sum_{k} b_{n}^{\dagger}(q+k) b_{n}(k)$. The Fermi velocities and interaction strengths for each component are renormalized by the inter-band forward scattering processes [Eqs. (8) and (9)] to

$$
\begin{aligned}
& v_{\rho, \sigma}=a\left[2 t_{\|} \pm\left(U_{\perp}+2 V_{\perp}\right) / 2 \pi\right] / \hbar, \\
& g_{\rho, \sigma}=a\left[4 V \pm\left(U_{\perp}+2 V_{\|}\right)\right],
\end{aligned}
$$

where the $+(-)$ signs above correspond to the $\rho(\sigma)$ label. We remind the reader that it is assumed throughout that the system of spinless fermions (5) is near half-filling. 
To proceed further, we first note that the pure Luttinger model (11) for the totalcharge excitations along the ladder corresponds precisely to the (Efetov-Larkin) hard-core boson mode ${ }^{19}$ for the rung hole pairs that is elaborated ref. 20 . The former is characterized entirely by the Luttinger liquid parameter $K_{\rho}=\left(2 \pi \hbar v_{\rho}-g_{\rho}\right)^{1 / 2} /\left(2 \pi \hbar v_{\rho}+g_{\rho}\right)^{1 / 2}$ that will re-appear below. The pseudo-spin piece (12) of the present spinless fermion description for a doped antiferromagnetic ladder is less trivial, however. Along the $S U(2)$-invariant line, $V_{\|}=V_{\perp}$, the pairing term $H_{\perp \text {,pair }}^{\prime}$ in Eq. (12) is null. Application of the bosonic representation ${ }^{14,15}$ for the spinless fermions then reveals that a gap, $\Delta_{\sigma} \neq 0$, opens in the spectrum of the pseudo-spin excitations (12) for $g_{\sigma} / a=2 V_{\perp}-U_{\perp}>0$ in the absence of transverse hopping, ${ }^{13} t_{\perp}=0$. For general inter-chain hopping, $t_{\perp} \neq 0$, and interactions, $V_{\|} \neq V_{\perp}$, it is instructive to move along the Luther-Emery "line" $g_{\sigma}=6 \pi \hbar v_{\sigma} / 5$, in which case the spinless fermions that correspond to the pseudo-spin system (12) are governed by the noninteracting Hamiltonian ${ }^{12}$

$$
\begin{aligned}
H_{\sigma}= & \hbar v_{F}^{\prime} \sum_{k} k\left(a_{k}^{\dagger} a_{k}-b_{k}^{\dagger} b_{k}\right)-2^{1 / 2} t_{\perp} \sum_{k}\left(a_{k}^{\dagger} a_{k}+b_{k}^{\dagger} b_{k}\right) \\
& +\Delta_{\sigma} \sum_{k}\left(a_{k}^{\dagger} b_{k}+\text { h.c. }\right)+\Delta_{\sigma}^{\prime} \sum_{k}\left(a_{k}^{\dagger} b_{-k}^{\dagger}+\text { h.c. }\right) .
\end{aligned}
$$

Here, the pseudo spin gaps have values $\Delta_{\sigma}=\left(a / \alpha_{0}\right)\left[\left(U_{\perp}-2 V_{\|}\right) / 2 \pi\right]$ and $\Delta_{\sigma}^{\prime}=\left(a / \alpha_{0}\right)\left(V^{\prime} / \pi\right)$ where $\alpha_{0}^{-1}$ is the momentum cutoff of the Luttinger model, while $v_{F}^{\prime}=\frac{4}{5} v_{\sigma}$. Consider first the $S U(2)$ invariant line $V_{\|}=V_{\perp}$, in which case the gap $\Delta_{\sigma}^{\prime}$ that originates from the pairing term (10) is null. The spinless fermions corresponding to the pseudo-spin sector therefore have energy eigenvalues $\varepsilon_{p}= \pm\left(v_{F}^{\prime 2} p^{2}+\Delta_{\sigma}^{2}\right)^{1 / 2}$. If $N_{n}$ denotes the number of spinless fermions in band $n$, then it follows that the band occupations are equal, $N_{+}=N_{-}$, for $t_{\perp}<\Delta_{\sigma} / 2^{1 / 2}$ and that $N_{+}-N_{-}=L \chi_{0}\left(t_{\perp}^{2}-\frac{1}{2} \Delta_{\sigma}^{2}\right)^{1 / 2}$ for $t_{\perp}>2^{-1 / 2} \Delta_{\sigma}{ }^{21}$ where $\chi_{0}=2 / \pi \hbar v_{F}^{\prime}$ is the pseudo-spin susceptibility. Band splitting is therefore absent below a critical inter-chain hopping matrix element. ${ }^{7}$ The general case, $\Delta_{\sigma}^{\prime} \neq 0$, off of the $S U(2)$ invariant line can also be analyzed at the Luther-Emery "line" (15). One finds that the product of all of the energy eigenvalues is $\Pi_{p>0}\left(v_{F}^{\prime 2} p^{2}+\Delta_{\sigma}^{2}+\Delta_{\sigma}^{\prime 2}\right)^{2}$, which never vanishes! We conclude that the net pseudo spin gap, $\Delta_{\text {charge }}=\left(\Delta_{\sigma}^{2}+\Delta_{\sigma}^{\prime 2}\right)^{1 / 2}$, is therefore robust with respect to $S U(2)$ symmetry breaking in the spinless fermion model (5).

Yet what is the physical character of the present ladder model at zero temperature? To answer this question, it is convenient to look again along the line, $V_{\|}=V_{\perp}$, in which 
case the $S U(2)$ non-invariant interaction term in the model Hamiltonian (5) is absent. Let us start by assuming no inter-chain hopping, $t_{\perp}=0$. Then the boundary at $U_{\perp}=2 \mathrm{~V}$ that marks the appearance of the pseudo spin gap $\Delta_{\sigma}$ can be identified with the phase boundary that exists between the staggered CDW state and the rung (hole-pair) CDW state in the strong-coupling limit (see Fig. 1 and ref. 22). A self-consistent calculation in terms of the CDW mean field $\left\langle R_{n}^{\dagger} L_{n}\right\rangle$ yields the approximate formula $\Delta_{\sigma}=\hbar \omega_{0} / \sinh \left(\pi \hbar v_{\sigma} / g_{\sigma}\right)$ for the pseudo-spin gap in the hole-pair regime, ${ }^{17} g_{\sigma}>0$, with prefactor $\omega_{0}=v_{\sigma} / \alpha_{0}$. It agrees reasonably well with the previous exact result along the Luther-Emery point. The same exact analysis indicates that band splitting generally remains absent at small enough inter-chain hopping amplitudes $t_{\perp}<\Delta_{\sigma} / 2^{1 / 2}$. We now address the initial question posed by computing the correlation functions at long distances and at long times within this hole-pair regime (see Table I). Then $S U(2)$ invariance yields the identity $\left\langle f_{0, l}(0) f_{i, l}^{\dagger}(t)\right\rangle=\left\langle f_{0, n}(0) f_{i, n}^{\dagger}(t)\right\rangle$ for the intra-chain one-particle propagator, where $\left\langle f_{0, n}(0) f_{i, n}^{\dagger}(t)\right\rangle=G_{R}(x, t)+G_{L}(x, t)$ is the propagator in the bonding/anti-bonding basis $(x=i a)$, with right and left moving components $G_{R}$ and $G_{L}$, respectively. Note that the latter independence of the spinless fermion propagation with the band index, $n$, is a result of the equal band occupation, $N_{+}=N_{-}$, present in the hole pair regime, $t_{\perp}<\Delta_{\sigma} / 2^{1 / 2}$. The application of the bosonization method plus pseudo spin-charge separation yields the forms $G_{R, L}=G_{R, L}^{(\rho)} \cdot G_{R, L}^{(\sigma)}$ for the right and left propagators, with a Luttinger liquid factor

$$
G_{R, L}^{(\rho)} \sim\left(x \mp v_{\rho}^{\prime} t\right)^{-1 / 2}\left[2 \pi \alpha_{0} /\left(x^{2}-v_{\rho}^{\prime 2} t^{2}\right)^{1 / 2}\right]^{\alpha_{\rho}}
$$

due to excitations of the total charge, ${ }^{14}$ and with pseudo-spin factor ${ }^{23}$

$$
G_{R, L}^{(\sigma)} \sim\left(x \mp v_{\sigma} t\right)^{-1 / 2} e^{-\left(\Delta_{\sigma} / \hbar v_{\sigma}\right)\left(x^{2}-v_{\sigma}^{2} t^{2}\right)^{1 / 2}}
$$

The exponent and velocity that appear in expression (16) have the forms $\alpha_{\rho}=\sinh ^{2} \psi_{\rho}$ and $v_{\rho}^{\prime}=v_{\rho} \operatorname{sech} 2 \psi_{\rho}$, respectively, with the hyperbolic angle $\psi_{\rho}$ set by the relation tanh $2 \psi_{\rho}=$ $-g_{\rho} / 2 \pi \hbar v_{\rho}$. A gap, $\Delta_{\text {charge }}=\Delta_{\sigma}$, therefore exists for one-particle charge excitations along chains. The inter-chain single-particle propagator, on the other hand, is by definition $\left\langle f_{0,1}(0) f_{i, 2}^{\dagger}(t)\right\rangle=\frac{1}{2}\left[\left\langle f_{0,+}(0) f_{i,+}^{\dagger}(t)\right\rangle-\left\langle f_{0,-}(0) f_{i,-}^{\dagger}(t)\right\rangle\right]$. Again, the band occupations $N_{+}$ and $N_{-}$are equal for $t_{\perp}<\Delta_{\sigma} / 2^{1 / 2}$, which implies that $\left\langle f_{0,1}(0) f_{i, 2}^{\dagger}(t)\right\rangle$ vanishes at long times and at long distances in such case. In other words, we find that coherent singleparticle tunneling of charge in between chains is entirely suppressed in the hole-pair regime. 
Notice that this is consistent with renormalization group calculations that find inter-chain hopping to be an irrelevant perturbation for Luttinger liquids with a pseudo gap in the density of states: ${ }^{24} N(\omega) \propto|\omega|^{\alpha}$, with exponent $\alpha>1$.

A similar analysis can be employed to obtain the static auto-correlators for various CDW and pair order parameters at long distance. These results are compiled in Table I. The intra-chain CDW correlator, for example, has the form ${ }^{12-15}\left\langle L_{l}^{\dagger}(0) R_{l}(0) R_{l}^{\dagger}(x) L_{l}(x)\right\rangle \sim$ $\cos \left(2 k_{F} x\right)\left(\alpha_{0} / x\right)^{K_{\rho}}$. It coincides with the form obtained for the density-density correlator of the hard-core boson model ${ }^{20}$ for the rung-hole pairs, ${ }^{19} b_{i}=f_{i, 1} f_{i, 2}$. On the other hand, both inter-chain CDW order and chain-hole-pair autocorrelations are short-range in the rung-hole-pair regimes, with a unique correlation length $\xi_{\sigma}=\hbar v_{\sigma} / 2 \Delta_{\sigma}$. Finally, the static autocorrelator for rung-hole pairs has the asymptotic form $\left\langle L_{1}(0) R_{2}(0) R_{2}^{\dagger}(x) L_{1}^{\dagger}(x)\right\rangle \sim$ $\left(\alpha_{0} / x\right)^{K_{\rho}^{-1}}$. This form also coincides with that obtained from the previously cited hardcore boson model for the propagation, $\left\langle b_{i} b_{j}^{\dagger}\right\rangle$, of rung-hole pairs. In conclusion, the phaseboundary separating dominant rung-CDW correlations from dominant rung-pair autocorrelations is evidently determined by the condition $K_{\rho}=1$ (see Table I). Given the absence of coherent tunneling that characterizes the rung-hole-pair regime in general, we interpret the latter phase $\left(K_{\rho}>1\right)$ as an IPT-type superconductor (see Fig. 1).

To address the question of the transverse conductivity of the ladder model (5), we shall now compute the transverse charge stiffness of the triangular three-leg ladder. ${ }^{8}$ In particular, consider the corresponding spinless fermion model [Eqs. (3) and $(4), N=3$ ] with a magnetic flux $\Phi$ threading each triangle formed by the rungs. Then along the line $V_{\|}=V_{\perp}$, the interaction terms of this model Hamiltonian are invariant with respect to $S U(3)$ rotations of the chain labels. After making algebraic manipulations similar to those employed to achieve the form (5) for the simple ladder, we obtain the form

$$
\begin{aligned}
H=\sum_{i}[ & -t_{\|} \sum_{l=1}^{3}\left(f_{i, l}^{\dagger} f_{i+1, l}+\text { h.c. }\right)-t_{\perp} \sum_{l=1}^{3}\left(e^{i \Phi / 3} f_{i, l}^{\dagger} f_{i, l+1}+\text { h.c. }\right)+ \\
& \left.+\left(U_{\perp}+2 V\right) \sum_{l=1}^{3} n_{i, l} n_{i, l+1}-\frac{1}{2} V\left(n_{i+1}-n_{i}\right)^{2}\right]
\end{aligned}
$$

for the triangular ladder model Hamiltonian, where $n_{i}=\sum_{l=1}^{3} n_{i, l}$ is the manifestly $S U(3)$ invariant number operator. After rotating to the basis $f_{i, 0}=3^{-1 / 2} \sum_{l=1}^{3} f_{i, l}$ and $f_{i, \pm}=3^{-1 / 2} \sum_{l=1}^{3} e^{ \pm i 2 \pi l / 3} f_{i, l}$ that diagonalizes the transverse kinetic energy, we obtain the 
previous Luttinger model [Eqs. (6), (7) and (9)], but with the band index summed over the new basis $n=0,+,-$. The new chemical potentials for each band are $\mu_{0}=2 t_{\perp} \cos (\Phi / 3)$ and $\mu_{ \pm}=2 t_{\perp} \cos [(\Phi \pm 2 \pi) / 3]$. Now consider the special line $U_{\perp}=-2 V$, along which the inter-band forward scattering interaction (9) vanishes. What remains is a generalized backscattering model with three internal quantum numbers. ${ }^{16}$ A mean-field analysis of this model ${ }^{17}$ finds that long-range CDW order of the type $\left\langle R_{n}^{\dagger} L_{n}\right\rangle$ is stable for effective attraction between rungs, $U_{\perp}<0$, with a single-particle gap $\Delta_{\sigma} \cong 2 \hbar \omega_{0} e^{-2 \pi t_{\|} / 3\left|U_{\perp}\right|}$, and prefactor $\hbar \omega_{0} \sim\left(a / \alpha_{0}\right) t_{\|}$. Hence for small enough inter-chain hopping, $t_{\perp}<\Delta_{\sigma} / 2$, the chemical potential of each band lies within the gap, which means that the transverse charge stiffness, $\partial^{2} E_{0} /\left.\partial \Phi^{2}\right|_{0}$, is null. In addition, since the band occupations, $N_{0}$ and $N_{ \pm}$, are all equal for $t_{\perp}<\Delta_{\sigma} / 2$, the single-particle intra-band amplitudes $\left\langle f_{0, n}(0) f_{i, n}^{\dagger}(t)\right\rangle$ are then all equal. The inter-chain single-particle amplitude $\left\langle f_{0, l}(0) f_{i, l+1}^{\dagger}(t)\right\rangle=\frac{1}{3} \sum_{n} e^{i 2 \pi n / 3}\left\langle f_{0, n}(0) f_{i, n}^{\dagger}(t)\right\rangle$ must therefore vanish as well, in agreement with the previous case of the simple ladder. On the basis of this mean-field analysis, ${ }^{17}$ we conclude that the present ladder model allows no coherent transport whatsoever in between chains in the (rung) hole-pair regime.

Let us now apply the spinless fermion model (5) to doped antiferromagnetic ladders by first drawing a comparison with the corresponding $t-J$ model. ${ }^{10}$ In the limit $\Delta_{\text {spin }} \rightarrow$ $\infty$ that is assumed throughout due to the relatively small energy scale, $t x$, for charge excitations, triplet excitations are forbidden. This means that the only possible charge carriers are hole pairs aligned parallel to the either the rungs or to the chains of the ladder (see Fig. 2). The spinless fermion system (5) describes the motion of these objects. Since rung hole pairs are responsible for coherent charge transport along the ladder, we have $t_{\|}=t$. In other words, the hopping of the spinless fermion along chains accounts for the effective tunneling of rung hole pairs between rungs. Yet what is the value of the interchain model parameter $t_{\perp}$ ? First, observe that the rotation of a rung hole pair into a chain hole pair is a two-stage process. As depicted in Fig. 2, the initial and final singlet pair states $(S=0)$ pass through an intermediate triplet pair state $(S=1)$ that is the lowest energy spin-excitation of the system. ${ }^{3,20}$ Second order perturbation theory then yields the matrix element $t_{\times}=t_{\|} t_{\perp} / \Delta_{\text {spin }}$ for the rotation of a rung hole pair into a chain hole pair, and vice-versa. Yet since such $90^{\circ}$ rotations represent the low-energy single-particle charge excitations of the ladder, we have the identity $t_{\times}=\Delta_{\text {charge }}$. This yields the expression 
$t_{\perp}=\left(\Delta_{\text {spin }} / t_{\|}\right) \Delta_{\text {charge }}$ for the effective inter-chain hopping matrix element. Yet since $t_{\|}=t$ and $\Delta_{\text {spin }}<J / 2$, we obtain the desired inequality

$$
t_{\perp}<\Delta_{\text {charge }} / 2
$$

for $J<t$. This indicates that the simple $t-J$ ladder is indeed consistently within the rung-hole pair regime per the spinless fermion description (5). Note also that (19) implies that coherent motion (11) of rung-hole pairs represents the only gapless charge excitation of the $t-J$ ladder. This is consistent with exact diagonalization results ${ }^{25}$ that find a singleparticle gap $\Delta_{\text {charge }}$ of order $\frac{3}{2} t x$ (the energy to $a d d$ an electron). ${ }^{20}$ The latter energy scale is small in comparison to the spin gap at low doping, $t x \ll J$, which justifies use of the spinless fermion model (5).

The remaining effective interaction parameters of the spinless fermion model (5) will be considered to be phenomenological. For simplicity, let us move along the $S U(2)$ invariant line $V_{\|}=V_{\perp}>0$. We then notably predict a phase transition in between a rung-CDW state and an IPT-type superconductor at $K_{\rho}=1$ (see Fig. 1). Comparison of the corresponding correlation exponents shown in Table I with those obtained from a density-matrix renormalization group analysis of the $t-J$ ladder $^{26}$ indicate that such a doped antiferromagnet is in the vicinity of this superconductor-insulator transition; i.e., $K_{\rho} \sim 1$. On this basis, we conclude that coherent single particle tunneling in between chains is absent in a lightly doped antiferromagnetic ladder, since $\Delta_{\sigma} \neq 0$ by Eq. (19). This does not exclude the possibility of (coherent) Josephson tunneling of hole pairs in between adjacent ladders, however. To address this issue, consider two neighboring doped ladders. Let us also suppose that adjacent ladders are shifted with respect to each other by half a lattice constant, which is in fact the case for real ladder systems that exhibit superconductivity. ${ }^{3,5,6}$ The dynamics of the rung-hole pairs, $b_{i}=f_{i, 1} f_{i, 2}$, is then equivalent to that of coupled spin- $\frac{1}{2} X X Z$ chains in magnetic field. ${ }^{19,20}$ The frustrating nature of the "zig-zag" (Josephson) coupling in between chains ${ }^{3}$ effectively reduces this system to isolated $X X Z$ chains, each with a renormalized intra-chain (Josephson) coupling. ${ }^{27} \mathrm{We}$ thus recover the previous superconductor/CDW transition, but with $K_{\rho}$ now dependent on the inter-chain Josephson coupling as well.

Concerning the experimental situation, the incoherent tunneling that is characteristic of the rung-CDW phase could explain the large conduction anisotropy seen in the normal 
state of antiferromagnetic ladder materials. ${ }^{4,9,28}$ In addition, if such a rung-CDW state were to be pinned, then all components of the resistivity tensor would exhibit insulating behavior in the low-temperature limit. This is indeed observed experimentally. ${ }^{4}$ It must be pointed out, however, that whether or not the low-temperature conductivity in doped antiferromagnetic ladder materials is intrinsic remains to be determined (see refs. 4, 9 and 28). If, on the other hand, the rung-CDW state would depin at some elevated temperature, then the generic Drude response characteristic of the present ladder model (5) would yield metallic behavior in the longitudinal resistivity. The latter is also observed in antiferromagnetic ladder materials at relatively high temperatures. ${ }^{4,28}$ Last, such materials are observed to go superconducting under extreme presure. ${ }^{5}$ A transition under pressure from a rung-CDW state to an IPT superconductor due to a strong dependence in the binding energy, ${ }^{10}-U_{\perp} \sim J$, of the rung-hole pairs with the lattice constants, for example, could account for this phenomenon. Very recently, however, the observation of high-temperature superconductivity in doped antiferromagnetic ladder materials at ambient pressure has been reported. ${ }^{6}$ The above discussion suggests that this system could transit into a rungCDW groundstate, on the contrary, by appropriate variations in the doping levels, or by varying other parameters like the pressure.

This work was supported in part by National Science Foundation grant No. DMR9322427. The author thanks G. Gomez-Santos, A. Leggett, D. Poilblanc, P. Sacramento, and V. Vieira for discussions. 


\section{References}

1. T. Ito et al., Nature (London) 350, 596 (1991).

2. J.M. Wheatley, T.C. Hsu, and P.W. Anderson, Phys. Rev. B 37, 5897 (1988); S. Chakravarty, A. Sudbø, P.W. Anderson, and S.P. Strong, Science 261, 337 (1993).

3. T.M. Rice, Z. Phys. B 103, 165 (1997).

4. N. Motoyama, T. Osafune, T. Kakeshita, H. Eisaki, and S. Uchida, Phys. Rev. B 55, R3386 (1997).

5. M. Uehara, T. Nagata, J. Akimitsu, H. Takahashi, N. Môri, and K. Kinoshita, J. Phys. Soc. Jpn. 65, 2764 (1996).

6. L. Leonyuk, G.-J. Babonas, R. Szymczak, H. Szymczak, M. Baran, A. Reza, V. Maltsev, L. Shvanskaya and V. Rybakov, Europhys. Lett. 45, 387 (1999).

7. D.G. Clarke, S.P. Strong, and P.W. Anderson, Phys. Rev. Lett. 72, 3218 (1994).

8. S. Capponi, D. Poilblanc, and F. Mila, Phys. Rev. B 54, 17547 (1996).

9. T. Osafune, N. Motoyama, H. Eisaki, and S. Uchida, Phys. Rev. Lett. 78, 1980 (1997).

10. E. Dagotto, J. Riera, and D.J. Scalapino, Phys. Rev. B 45, 5744 (1992); H. Tsunetsugu, M. Troyer and T.M. Rice, Phys. Rev. B 49, 16078 (1994).

11. J.P. Rodriguez, Bull. Amer. Phys. Soc. 43, 790 (1998).

12. A. Luther and V.J. Emery, Phys. Rev. Lett. 33, 589 (1974).

13. S.T. Chui and P.A. Lee, Phys. Rev. Lett. 35, 315 (1975).

14. V.J. Emery, in Highly Conducting One-dimensional Solids, ed. by J.T. Devreese, R.P. Evrard and V.E. van Doren (Plenum Press, New York, 1979).

15. J. Voit, Rep. Prog. Phys. 58, 977 (1995).

16. J.P. Rodriguez, Europhys. Lett. 39, 195 (1997).

17. J.P. Rodriguez, Phys. Rev. B 58, 944 (1998).

18. The intra-chain LE backscattering term (2) appears naturally in the weak-coupling analysis of the Hubbard ladder [see L. Balents and M.P.A. Fisher, Phys. Rev. B 53, 12133 (1996)].

19. K.B. Efetov and A.I. Larkin, Zh. Eksp. Teor. Fiz. 69, 704 (1975) [Sov. Phys. JETP 42, 390 (1976)].

20. M. Troyer, H. Tsunetsugu, and T.M. Rice, Phys. Rev. B 53, 251 (1996). 
21. V.L. Pokrovskii and A.L. Talanov, Zh. Eksp. Teor. Fiz. 78, 269 (1980) [Sov. Phys. JETP 51, 134 (1980)].

22. J.E. Hirsch, Phys. Rev. Lett. 53, 2327 (1984).

23. J. Voit, J. Phys.: Condens. Matter 8, L779 (1996).

24. D. Boies, C. Bourbonnais, and A.M.S. Tremblay, Phys. Rev. Lett. 74, 968 (1995).

25. C.A. Hayward, D. Poilblanc, Phys. Rev. 53, 11721 (1996).

26. C.A. Hayward, D. Poilblanc, R.M. Noack, D.J. Scalapino, and W. Hanke, Phys. Rev. Lett. 75, 926 (1995).

27. J.P. Rodriguez, P.D. Sacramento, V. Vieira, unpublished.

28. T. Osafune, N. Motoyama, H. Eisaki, S. Uchida and S. Tajima, Phys. Rev. Lett. 82, 1313 (1999). 
TABLE I. Listed is the correlation exponent $\eta$ obtained via the bosonization technique for various order parameters, $O(x)$, in the doped spin ladder model (5); i.e., $\left\langle O(x) O^{\dagger}(0)\right\rangle \propto$ $(\alpha / x)^{\eta}$. The $S U(2)$ - invariant case is assumed (see ref. 15). Below, the value $\eta=\infty$ indicates short-range order, while $K_{\rho}=\left(2 \pi \hbar v_{\rho}-g_{\rho}\right)^{1 / 2} /\left(2 \pi \hbar v_{\rho}+g_{\rho}\right)^{1 / 2}$.

\begin{tabular}{|c|c|c|c|}
\hline Order & Order Parameter & $\eta$ (Staggered CDW) & $\eta \quad$ (Rung Hole Pairs) \\
\hline CDW, $\|$ & $R_{l}^{\dagger} L_{l}$ & $K_{\rho}+1$ & $K_{\rho}$ \\
Pair, $\|$ & $R_{l} L_{l}$ & $K_{\rho}^{-1}+1$ & $\infty$ \\
CDW,$\perp$ & $R_{1}^{\dagger} L_{2}$ & $K_{\rho}+1$ & $\infty$ \\
Pair, $\perp$ & $R_{1} L_{2}$ & $K_{\rho}^{-1}+1$ & $K_{\rho}^{-1}$ \\
CDW $^{2}$ & $R_{1}^{\dagger} R_{2}^{\dagger} L_{2} L_{1}$ & $4 K_{\rho}$ & $4 K_{\rho}$ \\
Pair $^{2}$ & $R_{1} R_{2} L_{2} L_{1}$ & $4 K_{\rho}^{-1}$ & $4 K_{\rho}^{-1}$ \\
\hline
\end{tabular}




\section{Figure Captions}

FIGURE 1. The phase diagram of the $S U(2)$ - invariant model (5) for the charge excitations of a doped antiferromagnetic ladder is displayed in the regime of effective repulsion within each chain, $V_{\|}=V_{\perp}>0$, and in the absence of hopping in between chains. The rung hole-pair regime $\left(\Delta_{\sigma}>0\right)$ is, nevertheless, expected to persist for small enough inter-chain hopping matrix elements $t_{\perp} \lesssim \Delta_{\sigma} / 2$. In such case, the line separating dominant CDW correlations from superconducting ones among the rung-hole pairs is determined by the condition $K_{\rho}=1$.

FIGURE 2. A diagrammatic representation is given for the second-order matrix element $\left(t_{\times}\right)$that connects rung and chain hole pairs. The spin gap $\left(\Delta_{\text {spin }}\right)$ is presumed to be large in comparison to any difference in the binding energy $\left(\varepsilon_{\text {pair }}\right)$ between rung and chain hole pairs. 


$$
\mathrm{V}_{\|}=\mathrm{V}=\mathrm{V}_{\perp}
$$

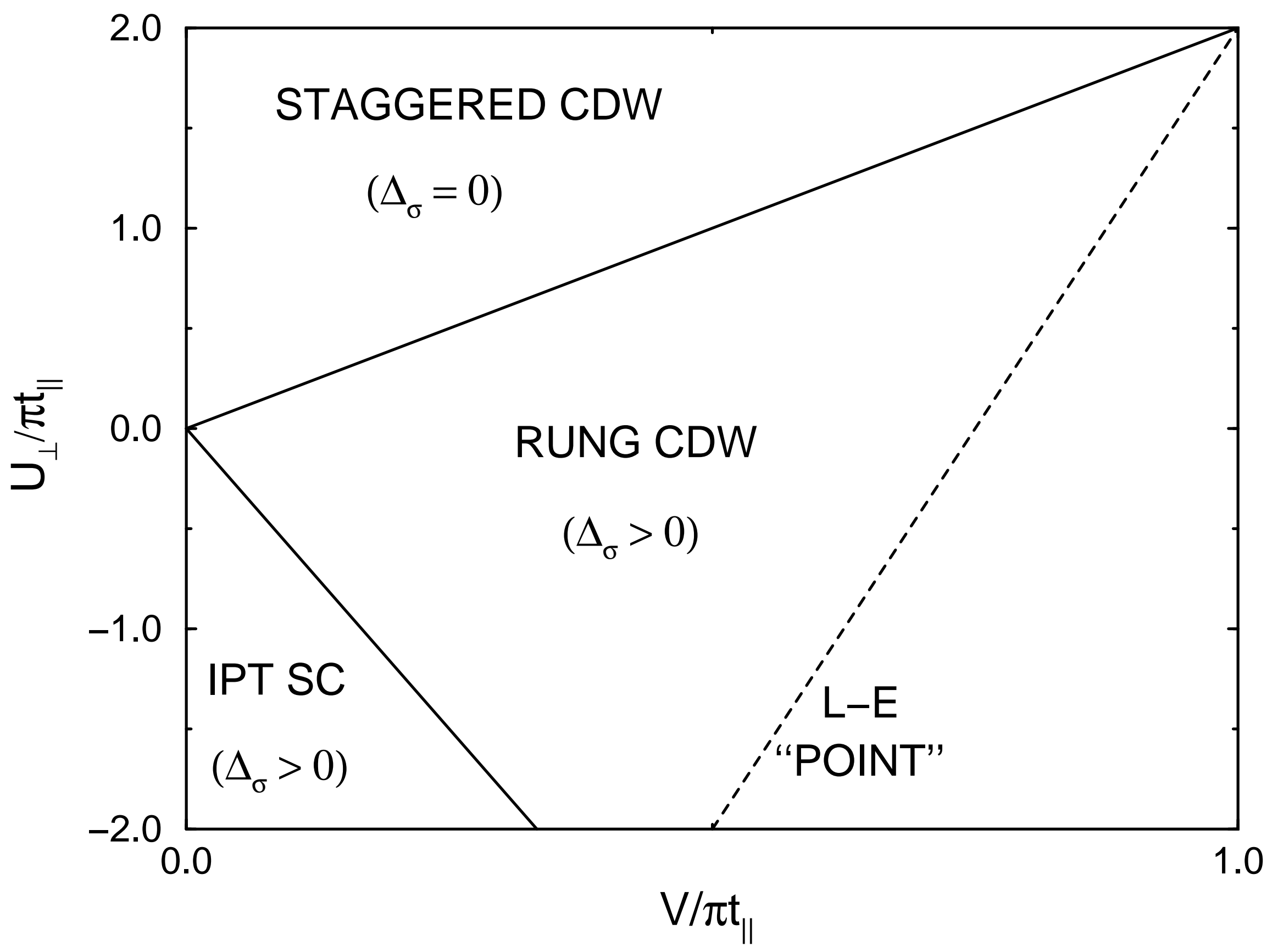




$$
\begin{aligned}
& (\mathrm{S}=0) \quad(\mathrm{S}=1) \quad(\mathrm{S}=0) \\
& \uparrow \downarrow \circ \downarrow \\
& \downarrow \uparrow \circ \uparrow \Rightarrow_{\downarrow \uparrow \circ \uparrow} \Rightarrow_{\downarrow \uparrow \downarrow \uparrow}{ }^{\uparrow \circ-\circ \downarrow} \\
& \left(\varepsilon_{\text {pair }}\right)\left(\varepsilon_{\text {pair }}+\Delta_{\text {spin }}\right) \quad\left(\varepsilon_{\text {pair }}\right)
\end{aligned}
$$

